\title{
The Influence of Nonuniform Reionization on the CMB
}

\section{Citation}

Zahn, Oliver, Matias Zaldarriaga, Lars Hernquist, and Matthew McQuinn. 2005. “The Influence of Nonuniform Reionization on the CMB." The Astrophysical Journal 630 (2): 657-66. https:// doi.org/10.1086/431947.

\section{Permanent link}

http://nrs.harvard.edu/urn-3:HUL.InstRepos:41381782

\section{Terms of Use}

This article was downloaded from Harvard University's DASH repository, and is made available under the terms and conditions applicable to Other Posted Material, as set forth at http:// nrs.harvard.edu/urn-3:HUL.InstRepos:dash.current.terms-of-use\#LAA

\section{Share Your Story}

The Harvard community has made this article openly available.

Please share how this access benefits you. Submit a story.

Accessibility 
Draft version OCtOBER 13, 2018

Preprint typeset using $\mathrm{LAT}_{\mathrm{E} X} \mathrm{X}$ style emulateapj v. 6/22/04

\title{
THE INFLUENCE OF NON-UNIFORM REIONIZATION ON THE CMB
}

\author{
Oliver Zahn ${ }^{1,3}$, Matias Zaldarriaga ${ }^{1,2}$, LARs Hernquist ${ }^{1}$ \& Matthew McQuinN ${ }^{1}$ \\ Draft version October 13, 2018
}

\begin{abstract}
We investigate the impact of spatial variations in the ionized fraction during reionization on temperature anisotropies in the CMB. We combine simulations of large scale structure to describe the underlying density field with an analytic model based on extended Press-Schechter theory to track the reionization process. We find that the power spectrum of the induced CMB anisotropies depends sensitively on the character of the reionization epoch. Models that differ in the extent of the "patchy phase" could be distinguished by future experiments such as the Atacama Cosmology Telescope (ACT) and the South Pole Telescope (SPT). In our models, the patchy signal peaks at $l \simeq 2000$, where it can be four times larger than the kinetic Sunyaev-Zel'dovich (kSZ)/Ostriker-Vishniac (OV) signal $\left(\Delta T_{\text {tot }} \simeq 2.6 \mu K\right)$. On scales beyond $l \simeq 4000$ the total Doppler signal is dominated by $\mathrm{kSZ} / \mathrm{OV}$, but the patchy signal can contribute up to $30 \%$ to the power spectrum. The effect of patchy reionization is largest on scales where the primordial CMB anisotropies dominate. Ignoring this contribution could lead to significant biases in the determination of cosmological parameters derived from CMB temperature measurements. Improvements in the theoretical modeling of the reionization epoch will become increasingly important to interpret the results of upcoming experiments.
\end{abstract}

Subject headings: cosmology: theory - cosmic microwave background - large-scale structure

\section{INTRODUCTION}

Cosmic Microwave Background (CMB) anisotropy experiments have now constrained temperature fluctuations down to scales of $4^{\prime}$ (Readhead et al. 2004; Holzapfel et al. 1999) resulting in a much improved understanding of inhomogeneities during the time of decoupling at $z \simeq 1100$ and of the global properties of the Universe. In combination with data from Supernovae Ia, measurements of the local expansion rate, galaxy clustering studies, and observations of the Lyman $\alpha$ forest, the CMB data has lead to the establishment of a "standard" cosmological model. We live in a Universe dominated by dark energy and with significantly more dark matter than baryons. Structure grew from a scale independent spectrum of primordial Gaussian fluctuations, in good agreement with predictions of inflationary models.

In the CMB community, theoretical and experimental interest is shifting to the study of secondary anisotropies on smaller angular scales. These are caused by fluctuations in the distribution of baryons and dark matter in the redshift regime $z \simeq 0-30$. Future data on the different secondary effects will constrain the way structure formation proceeded from the linear into the non-linear regimes. Secondary anisotropies can be divided into three categories: gravitational lensing effects, inverse Compton scattering of CMB photons by hot plasma, and Doppler related anisotropies.

Mass concentrations along the line of sight such as clusters, sheets, and filaments lead to weak deflection of the CMB photons, or Gravitational Lensing (for a review see chapter 9 of Bartelmann \& Schneider 2001). Gravitational lensing of the CMB can be used as a cos-

\footnotetext{
1 Harvard-Smithsonian Center for Astrophysics, 60 Garden Street, Cambridge, MA 02138, USA

2 Jefferson Physical Laboratory, Harvard University, Cambridge, MA 02138, USA

3 e-mail address: ozahn@cfa.harvard.edu
}

mological probe in various ways. The lensing effect on the CMB power spectrum could help break degeneracies between cosmological parameters (Metcalf \& Silk 1998; Stompor \& Efstathiou 1999). Lensing induces departures from Gaussianity on CMB maps that can be used to reconstruct the spatial distribution of the lensing mass (Zaldarriaga \& Seliak 1999; Hu \& Okamoto 2002). The CMB lensing signal can be correlated with galaxy lensing shear providing additional information. Thus, lensing can be used to probe the evolution of gravitational clustering, constrain the properties of the dark energy, the shape of the matter power spectrum, and neutrino physics (see e.g. Van Waerbeke et al. 2000; Hu 2002; Kaplinghat et al. 2003).

Inverse Compton scattering in the hot intracluster medium, also called the thermal Sunyaev-Zel'dovich effect (Zel'dovich \& Sunvaev 1969), changes the spectrum of the CMB photons, leading to cold spots (decrements) in the microwave background at frequencies below 217 $\mathrm{GHz}$ and to hot spots (increments) at frequencies above. The effect is proportional to the line-of-sight integrated pressure. Because it is independent of redshift, it is a unique probe of collapsing structures out to $z \simeq 3$ (see Figure (7). The thermal SZ effect has been measured in follow-up observations of X-ray clusters (see for instance Birkinshaw 1999; Carlstrom et al. 2000). Comparison of the cluster SZ temperatures with the X-ray measured temperatures leads to constraints on the angular diameter distance. The SZ effect also leaves a signature in the CMB power spectrum on small scales where the primordial CMB vanishes. BIMA (the Berkeley Illinois Maryland Association) and CBI (the Cosmic Background Imager) claim detections of this effect at $\geq 2 \sigma$ significance levels (Readhead et al. 2004; Holzapfel et al. 1999). CBI infers a LSS clustering amplitude on $8 \mathrm{Mpc} / \mathrm{h}$ scales of $\sigma_{8}=0.9$, which is at the upper level of constraints from cluster observations and weak lensing (for a recent compilation of experimental results see table 5 of 
Tegmark et al. 2004). Future large angular scale SZ surveys such as SPT promise to measure the cluster abundance as a function of mass and redshift, which will offer the possibility of constraining the matter density and the equation of state of the dark energy.

Finally, Doppler related effects are produced by the scattering of CMB photons off electrons moving as a result of the structure formation process. This process, also known as the kinetic Sunyaev-Zel'dovich effect when applied to clusters of galaxies (Sunvaev \& Zel'dovich 1980), leads to hot or cold spots, depending on whether the ionized baryons move toward or away from the observer. The frequency dependence of the photons is left unchanged, except for tiny relativistic corrections. These "Doppler" induced anisotropies are the only known way to measure the high redshift large scale velocity field.

Although our simulations account for each of these effects, our focus in this paper will be on the part of the Doppler effect generated during the epoch of reionization. This epoch is currently not well understood observationally.

The first year release of the WMAP data hinted at a large optical depth owing to reionization, $\tau_{r i}=0.17 \pm$ 0.05 (Spergel et al. 2003), through measurement of the "reionization bump" (Zaldarriaga 1997) in the temperature polarization cross correlation (Kogut et al. 2003). WMAP places only a weak constraint on the relative height of the acoustic oscillation peaks, so these data alone are not sufficient for constraining $\tau_{r i}$, because it is strongly degenerate with the tilt of scalar fluctuations $n_{s}$ and the baryon density parameter $\omega_{b}=\Omega_{b} h^{2}$. In combination with smaller scale CMB measurements, the data can be described by homogeneous reionization of the universe taking place at redshift $z \simeq 14 \pm 3$, or a Thomson scattering optical depth $\tau_{r i} \simeq 0.13 \pm 0.02$ (see e.g. Readhead et al. 2004; Bond et al. 2003). This estimate is also robust to the addition of other datasets to the analysis, such as the SDSS galaxy clustering survey (Tegmark et al. 2004) $\left(\tau_{r i}=0.124_{-0.057}^{+0.083}\right)$ or the Lyman alpha forest (Seliak et al. 2004) $\left(\tau_{r i}=0.133_{-0.045}^{+0.052}\right)$.

Since the large scale polarization measurements are sensitive only to the line-of-sight integrated free electron density, they do not require the time evolution of the ionized fraction to be a simple step function. Observations of the evolution of the Ly $\alpha$ optical depth in the absorption spectra of the highest redshift quasars at $z \simeq 6$ when combined with the CMB constraints favor a more complicated ionization history. The observations show a distinct Gunn-Peterson trough (Gunn \& Peterson 1965) and point to a rapidly evolving neutral fraction indicative of the final stages of reionization (Becker et al. 2001; Fan et al. 2001; White et al. 2003; Fan et al. 2004). Recently, there has also been an interpretation of the relatively high temperature of the Ly $\alpha$ forest at $z \simeq 2-4$ as evidence of an order unity change in the ionized fraction at $z<10$ (Theuns et al. 2002; Hui \& Haiman 2003), although this depends on the properties of He II reionization (Sokasian et al. 2002).

Numerical simulations of the star formation epoch have shown that a first generation of low metallicity (Population III) sources can survive negative feedback from the UV background they produce if shielded in massive halos for some time (Schaerer 2003; Bromm et al. 2002).
If they are heavy, these stars could produce an order of magnitude more ionizing photons per baryons than "normal" stars. Besides a more complex temporal behavior, the ionization fraction could have spatial variations; it could be "patchy" for a period of time while reionization is not complete. Naturally, we would like to have an observable that sheds light on the duration and structure of the reionization epoch.

The shape of the large scale polarization signal in the CMB could be used to constrain the evolution of the global ionization fraction, without resorting to information from secondary anisotropies (Zaldarriaga 1997; $\mathrm{Hu} \&$ Holder 2003). However, these scales are affected by sample variance limitations, so the available information is rather limited. Moreover, before such an approach can be successful, issues of foreground removal need to be clarified (Bennett et al. 2003; de Oliveira-Costa et al. 2004). In any event, large scale polarization measurements will not yield information about the morphology of reionization, as this is confined to sub-degree scales.

Several analytical models for patchy reionization have been presented in the literature. They vary significantly in the assumed size and time evolution of spherically shaped bubbles, and whether the ionized regions are correlated (Knox et al. 1998; Santos et al. 2003) or not (Gruzinov \& Hu 1998). Some models employ a prescription for correlating the ionizing sources by using the bias that can be calculated for the dark matter halos in which they presumably reside (Santos et al. 2003). All authors agree that on scales below $4^{\prime}$, where the primordial CMB signal falls rapidly owing to photon diffusion, the Doppler effect induced by patchiness could contribute enough to use it as a tool to study the reionization epoch.

Modeling patchy reionization more accurately requires the use of numerical simulations to incorporate effects of non-linear clustering and radiative transfer. The morphology of the ionized regions depends sensitively on the star formation model (see e.g. the reionization study companion by Barkana \& Loeb 2001), source properties, feedback processes, and radiative transfer (these effects have for example been modeled in Abel \& Wandelt 2002; Gnedin \& Jaffe 2001; Ciardi et al. 2003; Sokasian et al. 2003, 2004). These simulations have given us evidence that reionization was much less homogeneous than previously thought (Miralda-Escudé et al. 1998; Barkana \& Loeb 2001). Reionization tends to proceed from high to low density regions (Sokasian et al. 2003, 2004; Ciardi et al. 2003) and recombinations seem to play a role subdominant to large scale bias. Strömgren spheres of neighboring protogalaxies overlap, and overdensities will harbor large ionized regions. Because HII regions extend to larger radii than the correlation length of galaxies, building an analytic model solely on local galaxy properties appears to be difficult. Besides uncertainties over the physics in current radiative transfer calculations of reionization, memory and CPU requirements pose a serious limitation, so that these simulations have so far only been performed as a post-processing step on scales of up to $10 \mathrm{Mpc} / \mathrm{h}$, corresponding to $\simeq 6 \mathrm{ar}$ cminutes on the sky (Furlanetto et al. 2004a). If the HII regions are of comparable size, sample variance becomes a problem for these simulations.

In this paper, we use a hybrid approach between an analytic model for the formation of HII regions, and 
smoothed particle hydrodynamics (SPH) simulations of large scale structure. The advantage over analytic models of patchy reionization is that our sources follow the complex clustering behavior of dark matter and baryons. An advantage over currently feasible full radiative transfer calculations of the reionization epoch is that we can investigate the morphological properties of reionization on scales an order of magnitude larger with rather small memory and CPU requirements. Our prescription does not directly address many of the (uncertain) physical details of reionization related to star formation, feedback processes, clumpiness, recombinations, and radiative transfer. However, by combining these properties into a single parameter, we are able to explore the basic parameter dependencies, and we believe it is a good starting point to help us understand the morphological properties of cosmological reionization and its influence on the CMB.

We will describe the analytic model for the morphology of patchy reionization suggested by Furlanetto et al. (2004b) in the next section. In this description, the size distribution of HII regions is derived in a way analogous to the Press-Schechter formalism for the halo mass function, so that sources are biased towards large scale matter concentrations. The model has only one free parameter, the efficiency of the first sources to ionize the surrounding medium.

In Section 3 we describe our implementation of the model into SPH simulations as a post-processing step. Since the costs in memory and CPU consumption in this scheme are relatively low, one can imagine testing a wide array of behaviors for the ionization efficiency of the first sources against experiments.

One of the parameterizations we employ, in Section [4 attempts to do justice to the apparently contradicting measurements of a large integrated Thompson optical depth in CMB polarization measurements that indicates an onset of reionization at redshifts beyond $z=$ 15 , and a rapid decline of the neutral fraction around $z=6-7$, seen in measurements of the Gunn-Peterson optical depth by measuring spectra of distant quasars. We model this by assuming two succeeding reionization epochs, in which HII regions first are produced by low metallicity (Pop. III) stars, and later expand further during an epoch dominated by Pop. II stars, so that on the whole patchiness lasts longer.

In Section 5 we investigate whether the next generation of ground based bolometric arrays will be able to measure reionization and distinguish between different scenarios. The specifications of the Atacama Cosmology Telescope (ACT) ${ }^{4}$ (Kosowskv 2003) and the South Pole Telescope $(\mathrm{SPT})^{5}$ (Ruhl et al. 2004) will be used to predict how well these experiments can measure CMB temperature power spectra in the region where secondary effects dominate over primordial CMB anisotropies. We find that $\mathrm{ACT} / \mathrm{SPT}$ should be able to distinguish between sudden homogeneous reionization and patchy reionization at a high level of significance, even if the total optical depth in both scenarios were the same.

Since the patchy signal peaks on scales where the primordial anisotropy dominates, cosmological parameter

\footnotetext{
${ }^{4}$ see http://www.hep.upenn.edu/ angelica/act/act.html

${ }^{5}$ see http://astro.uchicago.edu/spt/
}

estimation may become biased. We will show in Section 5.2 that precision experiments such as Planck should take this bias into account either by focusing on their polarization data or by adding a reionization parameter to their analysis.

Our numerical simulations of large scale structure and the power spectra we used to generate realizations of the primordial CMB assume a cosmology in agreement with WMAP constraints: $\Omega_{d m}=0.26, \Omega_{\Lambda}=0.7, \Omega_{b}=0.04$, fluctuations on $8 \mathrm{Mpc} / \mathrm{h}$ scales of $\sigma_{8}=0.9$, and no tilt $\left(n_{S}=1\right)$ or running $\left(\alpha_{S}=0\right)$ of the spectral index.

\section{ANALYTIC MODEL FOR PATCHY REIONIZATION}

We review the model for the growth of HII regions during reionization proposed by Furlanetto et al. (2004b). The standard way to describe ionization of the IGM is to associate an HII region with a single galaxy. The size distribution of ionized regions then follows directly from the halo mass function when the ansatz $m_{i o n}=\zeta m_{g a l}$ is made, where $m_{\text {gal }}$ is the mass in a collapsed object. The parameter $\zeta$ is the efficiency factor for ionization, for example composed as $\zeta=f_{\text {esc }} f_{*} N_{\gamma / b} n_{\text {rec }}^{-1}$, where $f_{\text {esc }}$ is the escape fraction of ionizing photons from the object, $f_{*}$ is the star formation efficiency, $N_{\gamma / b}$ the number of ionizing photons produced per baryon by stars, and $n_{r e c}$ is the typical number of times a hydrogen atom has recombined. The efficiency factor is a rough combination of uncertain source properties, but encapsulating a variety of reionization scenarios in it can be regarded as a starting point to gain insights in the morphological properties of the partly ionized phase.

According to the extended Press-Schechter model (Bond et al. 1991), the collapsed fraction (or the fraction of baryons that lie in galaxies) in a region of size $r$ depends on the mean overdensity of that region, $\bar{\delta}_{r}$, as

$$
f_{\text {coll }}\left(m_{\text {min }}\right)=\operatorname{erfc}\left[\frac{\delta_{c}(z)-\bar{\delta}_{r}}{\sqrt{2\left[\sigma^{2}\left(r_{\text {min }}\right)-\sigma^{2}(r)\right]}}\right] .
$$

Here, $\sigma^{2}(r)$ is the linear theory rms fluctuation on scale $\mathrm{r}$ and $r_{\text {min }}$ is taken to be the radius that encloses the mass $\mathrm{m}_{\min }$ (at average density $\bar{\rho}$ ) corresponding to a virial temperature of $10^{4} \mathrm{~K}$, at which atomic hydrogen line cooling becomes efficient and $\delta_{c}(z)$ is the numerical factor 1.686 scaled to today using linear theory. The redshift dependence of the minimum mass (see Barkana \& Loeb 2001) can be described by

$$
m_{\min } \simeq 3.0 \times 10^{9}(1+z)^{-1.5} M_{\odot} .
$$

The mass in galaxies that can create enough luminous sources to fully ionize all hydrogen atoms is inversely proportional to the ionizing efficiency, so one requires that

$$
f_{\text {coll }} \geq \zeta^{-1} \text {. }
$$

Hence, we can define a barrier which fluctuations have to cross for their baryonic content to become ionized:

$\delta_{r} \geq \delta_{x}(m, z) \equiv \delta_{c}(z)-\sqrt{2} \operatorname{erfc}^{-1}\left(\zeta^{-1}\right)\left[\sigma^{2}\left(r_{\min }\right)-\sigma^{2}(r)\right]^{1 / 2}$.

Because it assumes Gaussian fluctuations on the mass scale $m$, this formalism can be applied only to mass scales larger than the typical size of collapsed objects. 


\section{SIMULATION OF PATCHY REIONIZATION}

Our approach is to use large scale simulations of the cosmic web as a basis for applying the model described in the last section as a post-processing step to generate HII regions during the reionization phase. The underlying large scale structure was simulated using the parallel Tree-PM/SPH solver GADGET (Springel, Yoshida \& White 2001), based on the fully conservative implementation of SPH by Springel \& Hernquist (2002). Here, we used the results of runs with a boxsize of $100 \mathrm{Mpc} / h$ and $216^{3}$ particles, with parameters corresponding to the G-series runs of Springel \& Hernquist (2003). In what follows, we employ a simulation that included only "adiabatic" gas physics; i.e. the gas can heat or cool adiabatically and be shocked, but we do not include radiative effects or the consequences of star formation and associated feedback processes. Snapshots of the simulation are produced every light crossing time interval. This leads to 77 outputs between $z=0$ and $z=20$. These simulations are described in more detail in White et al. (2002).

To generate inhomogeneously ionized regions according to the model described in Section [2] we discretize the matter fluctuations in our simulation boxes into $256^{3}$ cells. The overdensity, $\delta$, is smoothed with a top hat window function.

To find the smoothing scale at which a given cell is ionized we have to keep in mind that it could also be ionized by photons originating from neighboring regions. Thus we should smooth the density on all possible scales to see whether a given point was above the ionization threshold of equation (4) for some smoothing scale. In practice, we start at large radii (comparable to the simulation box size) and record the smoothed overdensity as we smooth logarithmically on progressively smaller scales. When a cell first crosses the barrier (which depends only on redshift and ionization efficiency), it is deemed ionized. If later, as a function of decreasing scale, it crosses the barrier downwards, this means that the region had been initially ionized by a neighboring overdensity.

Figure 1 shows the random walk of $\delta_{0}$, the density fluctuation scaled to the present using linear theory, for three different regions inside our box (at redshift $z=14.2$ ) with increasing $\sigma^{2}(m)$ (decreasing smoothing radius $r$ ). It also shows the ionization efficiency dependent barrier, from equation (4), as the solid curve. The short-dashed curve describes a region of high overdensity that selfionizes. In the dotted curve at $\sigma^{2}(m) \simeq 2.2$ the barrier is crossed downwards, so the volume element was ionized by sources in neighboring cells. The long-dashed curve corresponds to an element that did not ionize at this redshift.

To sample a wide range of bubble sizes, and obtain a smoothly varying ionization fraction, we varied the barrier in radial direction throughout each box by adjusting the redshift dependent minimal ionization radius. The reionization information for each of the $256^{3}$ cells is stored. A $8 \mathrm{Mpc} / \mathrm{h}$ deep cut through the stacked outputs between $z \simeq 12$ and $z \simeq 17$ is shown in Figure 2] for one of the models we will describe in the next section, in which the ionization efficiency is constant, $\zeta=60$. The periodic boundary conditions of our simulation boxes are apparent in this plot.

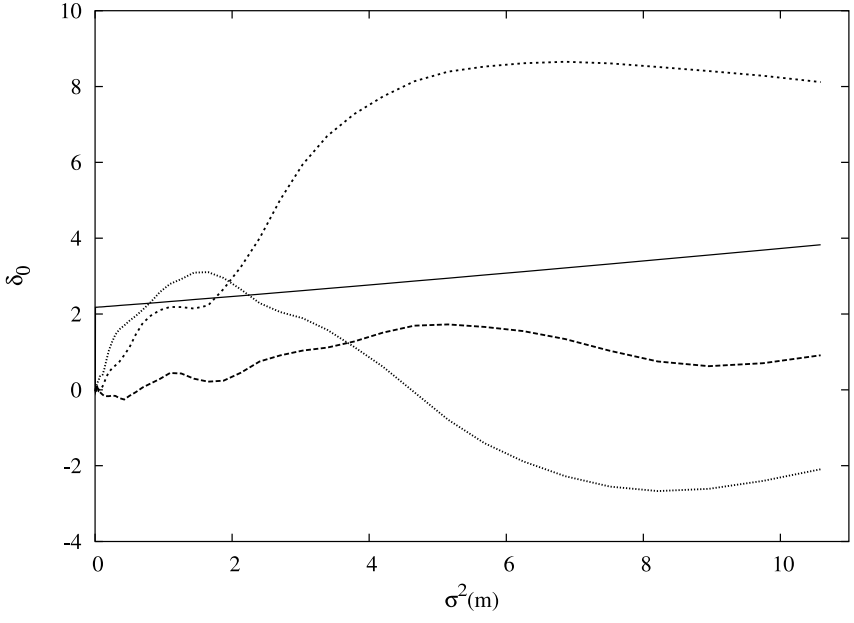

Fig. 1. - The behavior of the overdensity scaled to today, $\delta_{0}$, for four regions from our $256^{3}$ calculation. The abscissa in this plot is the rms fluctuation, dependent on the applied smoothing scale. The solid curve is the barrier given from equation (4. The region corresponding to the short dashed curve crosses the barrier for ionization at $\sigma^{2}(m) \simeq 1.8$. The region described by the dotted curve wanders below the barrier again, but has been ionized by sources in a neighboring region with higher density. Finally the long dashed curve represents a region in which the gas stays neutral at this particular redshift $(\bar{z}=14.2)$.

To compute the electron scattering and gravitational lensing effects from the content of the simulation boxes, we only need to store two-dimensional maps of the product of pressure and volume (Compton scattering), the velocity weighted free electron density (Doppler effect), and the matter density (lensing). We simulate regions on the sky with an angular extent of $1^{\circ}$ each, at a resolution of $256^{2}$ pixels. In the small angle approximation we can simply project the content of each simulation box onto a plane in its center. At high redshifts, $z>5.7$, the field of view exceeds the boxsize, so we use the periodic boundary conditions to cover it. During the patchy regime, only those gas particles that are located in an ionized cell according to the information we stored earlier contribute to the signal. At later times all particles are assumed ionized. We translate and rotate the positions and velocities of the particles randomly before doing the projection onto the plane. The gas particle properties are distributed over surrounding pixels weighted by the SPH kernel. For dark matter particle masses we use the cloudin-cell algorithm. Photons are traced through the planes from a regular grid close to the observer towards the last scattering surface. We produced Gaussian random fields of the primordial CMB using spherical harmonic decomposition coefficients generated with the publicly available code CMBFAST (Seliak \& Zaldarriaga 1996) ${ }^{6}$.

In models of patchy reionization, $\mathrm{CMB}$ anisotropies are caused by two types of contributions induced by Doppler scatterings: perturbations in the baryon density $\omega_{\mathrm{b}}$, given by $\delta \omega_{\mathrm{b}}=\rho_{\mathrm{b}} / \rho_{\text {tot }}-1$, and local changes in the ionization fraction $\delta x_{e}$. These produce a change

\footnotetext{
6 http://www.cmbfast.org
} 


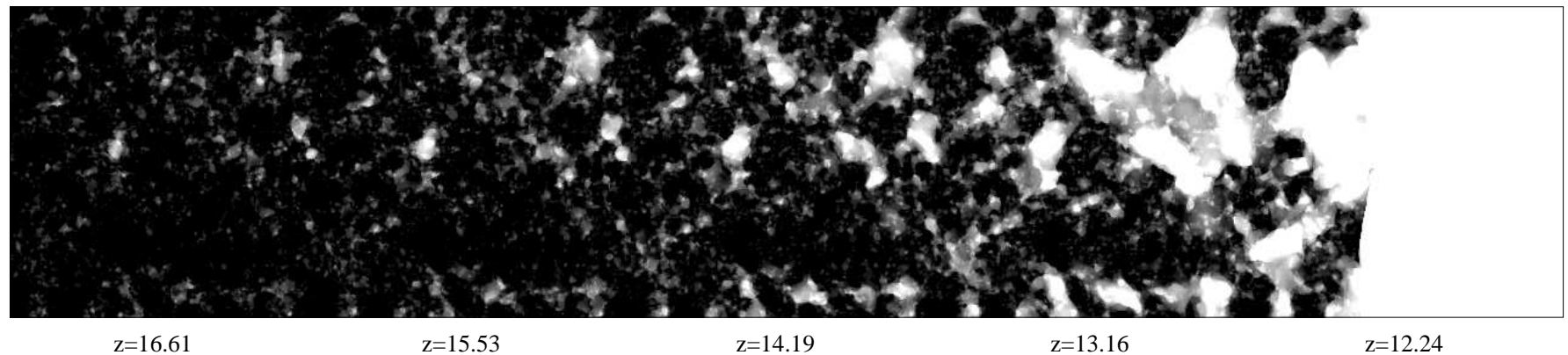

FIG. 2.- Time development between redshifts $z=12$ and $z=17$ of an $8 \mathrm{Mpc} / \mathrm{h}$ deep slice through an inhomogeneously ionized $\zeta=60$ (constant) box, with increasing ionization fraction. The outputs with periodic boundary conditions are simply stacked behind each other here. In order to obtain realistic maps, the individual outputs where randomly rotated and translated in our simulations. At the low redshift end, the bubbles become comparable in size to the box.

in temperature of the CMB blackbody. The total contribution to the temperature anisotropy is given by the integral over conformal time ${ }^{7}$

$$
\frac{\Delta T_{k S Z}}{T_{C M B}}(\hat{\mathbf{n}})=-\frac{\sigma_{T}}{c} \bar{n}_{p}\left(\eta_{0}\right) \int d \eta\left[a^{-2} e^{-\tau_{r i}(\eta)} \bar{x}_{e}(\eta)\right] \hat{\mathbf{n}} \cdot q
$$

with

$$
q=\left(1+\frac{\delta x_{e}}{\bar{x}_{e}(\eta)}\right)\left(1+\delta \omega_{\mathrm{b}}\right) \mathbf{v} .
$$

The Thomson scattering optical depth is $\sigma_{T}, \tau_{r i}$ is the optical depth from the observer to conformal time $\eta$, and $\hat{\mathbf{n}}$ is the line of sight unit vector.

To improve our understanding of reionization, various parameterizations of $\zeta$ and its derivatives may be compared to data from the next generation of experiments. For each redshift, the only free parameter in our model of patchy reionization is the efficiency of the first sources which ionize the surrounding intergalactic medium. In contrast to radiative transfer simulations, our implementation is not limited by CPU or memory related problems. For each box we need to Fourier transform $\simeq 50$ times back and forward to do the smoothing. This takes $\simeq 100$ minutes per model on a Xeon $3.2 \mathrm{GHz}$ computer. The implementation may be parallelized easily, hence the realization of a large number of parameterizations of $\zeta$ is feasible.

Even in the context of our simplified model, there are two additional limitations to the accuracy of our present simulations. First, because the mean overdensity on scales larger than the simulation box is artificially set to zero in numerical simulations, in order to achieve periodic boundary conditions, we expect a systematic bias in the overall ionization fraction. Barkana \& Loeb (2004) estimated this effect and showed that it should be less than $1 \%$ in simulations of $100 \mathrm{Mpc} / \mathrm{h}$ size. A different bias arises from our finite mass resolution. Our lack of structure on scales smaller than $L_{b o x} /\left(N_{\text {part. }}\right)^{1 / 3}$ leads to a slight delay in the onset of reionization. Very small overdensities on scales below $0.5 \mathrm{Mpc} / \mathrm{h}$, that could harbor ionizing sources with HII regions around them, are not captured by our analysis.

\footnotetext{
7 During homogeneous reionization and at lower redshifts, where galaxy clusters are present, this is traditionally called the kinetic Sunyaev-Zel'dovich effect, at higher redshifts the Ostriker-Vishniac (Ostriker \& Vishniad 1986) effect
}

\section{RESULTS FOR DIFFERENT TIME DEPENDENCE OF THE IONIZATION EFFICIENCY}

In our analysis we will compare three different reionization scenarios. These are tuned such that they all lead to a comparable integrated optical depth, $\tau_{r i} \simeq$ $0.125-0.133$. The different histories of the fraction of ionized volume elements $Q(z)$ are shown in Figure 3

Model A describes a universe that undergoes homogeneous and instantaneous reionization at redshift 14 . This is the standard scenario (Seliak \& Zaldarriaga 1996; Lewis et al. 2000) assumed in likelihood analyses of large scale CMB polarization anisotropy (in its correlation with temperature, see Kogut et al. 2003; Spergel et al. 2003). This model has $\tau_{r i}=0.133$.

Model B is the patchy model described above, with the assumption of constant source properties. An ionization efficiency $\zeta=60$ leads to reionization beginning at $z=19$ and concluding by $z=12$. This model has $\tau_{r i}=0.130$.

Model C exhibits extended patchiness. We describe this by assuming a first generation of metal free sources starting at redshift 20 with an ionization efficiency of $\zeta=200$ (it has been suggested that their photon output could be 10-20 times higher than that of normal stars, see Schaerer 2003; Bromm et al. 2002; Somerville et al. 2003). In our model, these sources sustain themselves only to redshifts around 15, because their hard photons dissociate fragile $\mathrm{H}_{2}$ they need for cooling (e.g. Mackev et al. 2003; Yoshida et al. 2003, 2004). However, the first sources are assumed to leave pockets of ionized medium which begin to harbor Pop II stars. The initial mass function becomes less top heavy with time, and we describe the resulting Population II stars with an efficiency $\zeta=12$ (see, e.g. Furlanetto et al. 2004c). This leads to a total optical depth of $\tau_{r i}=0.125$. Our model has a monotonically evolving ionization fraction. Double reionization scenarios with an intermittent decrease of the ionization fraction have for instance been suggested in Cen (2003) and Wvithe \& Loeb (2003) but seem difficult to realize in practice (Furlanetto \& Loeb 2004). We note that another way of reconciling CMB with quasar spectra observations is by resorting to recombinations that leave $0.1 \%$ of the universe neutral until $z=6-7$ (Sokasian et al. 2003, 2004).

In Figure 3 we plot for all three models the fraction of ionized volume elements as a function of redshift, $Q(z)$. 


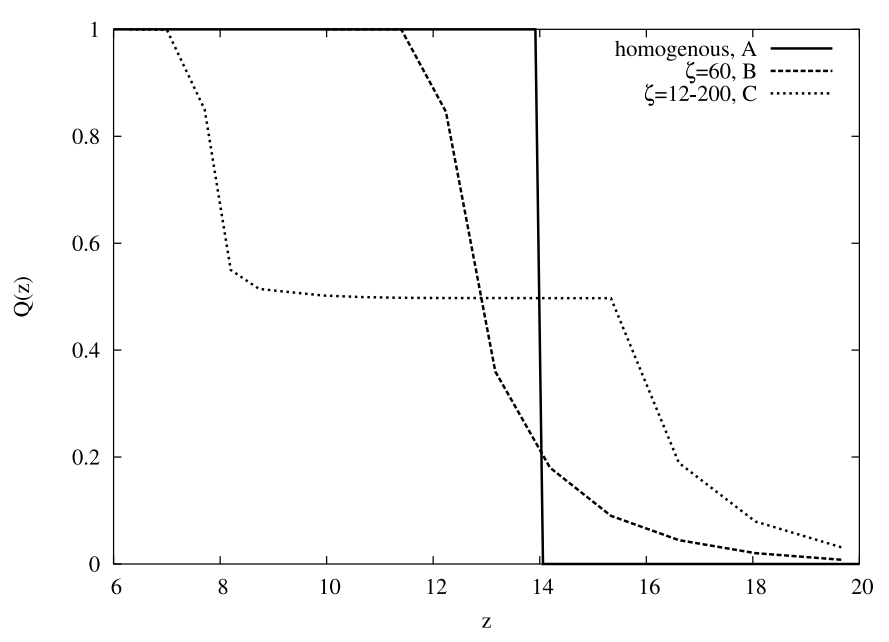

FIG. 3.- Evolution of the fraction of ionized volume elements $Q(z)$ in our calculations of reionization. Solid curve corresponds to instantaneous reionization at redshift $z=14$, yielding an optical depth of $\tau_{r i} \simeq 0.125$ for standard cosmological parameters. The dashed curve represents our patchy model in the case of a constant ionizing efficiency of sources. The dotted curve is for a model where a first generation of metal-free stars can survive the negative feedback of $\mathrm{H}_{2}$ photodissociation for a limited period of time, then formation of these stars comes to a halt, leaving a network of HII regions. After a while, Population II stars are born inside these regions and their photons gradually lead to a homogeneously ionized universe (Model C). Model C spends the longest time in the partly ionized regime and will therefore lead to the largest signal of the three models which have comparable total optical depth.

In Model A, the neutral fraction drops instantaneously at $z=14$, in Model B sources of a constant ionization efficiency $\zeta=60$ lead to a brief partly ionized phase, and in Model C the ionization fraction "freezes in" for some time, while metal free Pop. III stars cease to exist and leave residual ionized bubbles for "normal" Pop. II stars to be created and to ionize the medium fully at $z=7-8$.

We divide the total Doppler signal into three different redshift regimes in Figure 4 The maps have a side length of $1^{\circ}$ and are smoothed with a Gaussian beam of width $\theta \simeq 1^{\prime}$, corresponding to a multipole number of $l_{\max } \simeq 10000$, comparable to the angular resolution of $\mathrm{ACT}$, and slightly below that of SPT. It is expected that during the interval from $z=0$ to $z=3$, more strongly clustered regions make the Doppler effect maps highly non-Gaussian. We show this by the solid line (PDF) in Figure 5 In the picture in the center $(z=3-11)$ structure formation is less advanced. The corresponding histogram can be well-approximated by a Gaussian, as shown by the long-dashed curve in Figure 5 Signals created by radially moving inhomogeneities during this epoch are referred to as the Ostriker-Vishniac effect (Ostriker \& Vishniad 1986). The plot on the right of Figure 4 is the patchy epoch of reionization Model B. Again, this epoch has a largely Gaussian morphology (short-dashed line of Figure [5), because structures on scales of the ionized bubbles are just beginning to collapse. According to this figure, large bubbles form on scales of tens of Mpc. The redshift regimes represented by the left and middle picture have the same properties in all three of our reionization scenarios.

The upper panel of Figure [6 shows that in the homogeneously reionizing universe, the largest portion of the Doppler signal comes from low redshifts, the kSZ regime. The upper panel represents the case of homogeneous reionization, Model A. Contrary to the thermal SZ effect, the signal still carries information about higher redshifts, $z \leq 10$. We can use this principle to gain knowledge about the details of reionization taking place at high redshifts. The additional contributions from larger redshifts $(z>10)$ in Model $\mathrm{C}$ are shown in the lower panel of the figure.

For comparison, we show in Figure 7 how different redshift regimes contribute to the thermal SZ effect (where $\Delta T / T=-2 y$ for low frequencies). The plot shows that this signal is basically saturated at $z=3$; the thermal SZ effect receives most of its contribution from galaxy clusters in the more nearby universe.

\section{OBSERVABILITY OF PATCHY REIONIZATION WITH FUTURE CMB EXPERIMENTS}

In this section, we assess the observability of patchy reionization with future experiments. $\mathrm{ACT}$ and $\mathrm{SPT}$ should be able to distinguish different reionization scenarios with high significance by measuring temperature power spectra. In their cosmological parameter analyses, all-sky experiments such as Planck will have to account for patchy reionization as a possible source of bias and need to rely on their polarization data in order to obtain proper constraints.

\subsection{Power spectral constraints from $A C T$ and $S P T$}

The effect of patchy reionization on the CMB power spectrum is of similar magnitude to that of the Doppler effect induced by variations in the mean density. The first source population is heavily biased so the patchy reionization signal peaks on larger angular scales. Figure 2 shows that for the regime in which the ionization fraction is roughly $50 \%$ (which is where most of the signal comes from) the bubbles reach sizes of tens of comoving Mpc. In Figure 8 we plot the different contributions to the Doppler power spectrum. Patchy reionization in Model $\mathrm{C}$ with $\zeta=12-200$ lasts from redshifts 20 to 7. The signal imprinted in the CMB from this epoch is shown in solid. At later times, the universe is homogeneously reionized and the dashed line shows the kinetic SZ effect for this period.

Inverse Compton scattering in galaxy clusters leads to a larger signal than that caused by Doppler scattering. Because only a small fraction of the CMB photons present are likely to scatter inside the cluster medium ( $\simeq 1 \%$ for a massive $15 \mathrm{keV}$ cluster), the distribution will not thermalize to that of the hot gas. The effect has a characteristic frequency dependence, and it can be distinguished from Doppler scatterings which leave the frequency distribution of the photons unchanged. The dot-dashed line in Figure 8 is the combination of the secondary signal with the primordial CMB, once the thermal SZ with its characteristic frequency distribution has been removed.

On scales where patchiness during reionization contributes more to the total signal than the Doppler effect owing to modulations in the density, $(l<4000)$, the primordial anisotropies dominate. To study the secondary 

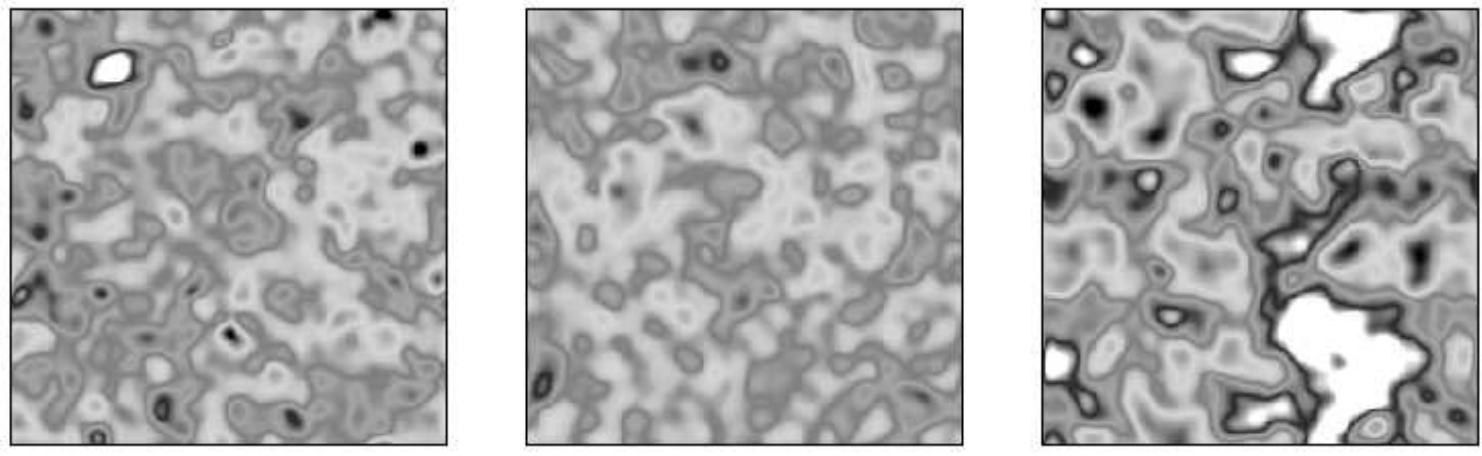

FIG. 4.- Based on the implementation of Model B with a constant value of $\zeta$, we divide the Doppler signal into three redshift epochs. Plot on the left shows the kinetic Sunyaev-Zel'dovich effect out to redshift 3, in the middle the Ostriker-Vishniac regime from $z=3$ out to $z=11$ is shown, and on the right the regime of non-uniform (patchy) reionization. Each picture shows the same angular extent, $1^{\circ}$. The left panel exhibits highly clustered structures. With increasing redshift, the clustering becomes more linear and patchy reionization leads to CMB signals on large scales. We smoothed the maps obtained from our simulations by a Gaussian beam corresponding to $l=10000 \simeq 1^{\prime}$, comparable to the angular resolutions of ACT and SPT.

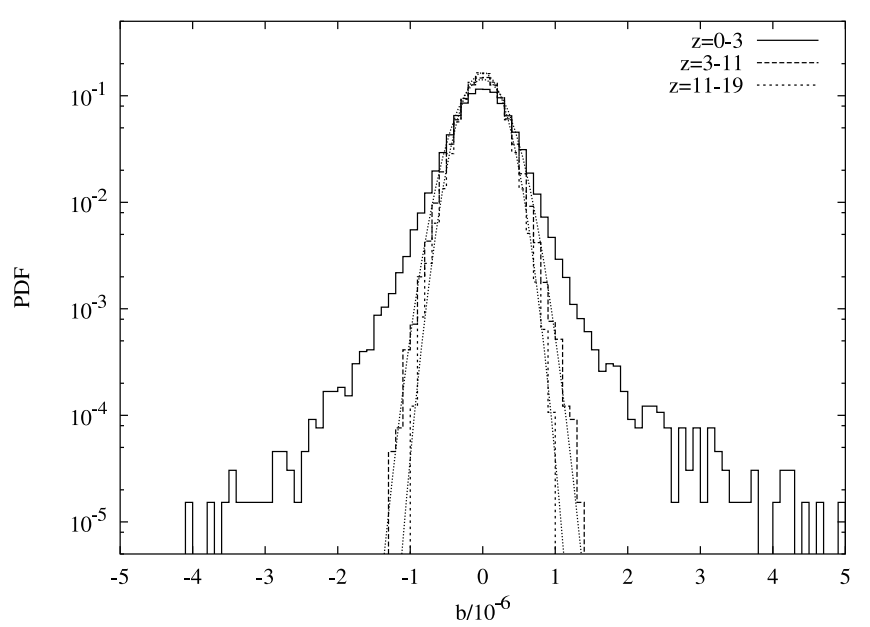

FIG. 5.- The probability distribution functions for the three maps in Figure 4 are shown. The solid line is for the kinetic SZ regime $\mathrm{z}=0-3$, the long-dashed line for the Ostriker-Vishniac era $\mathrm{z}=3-11$, the short-dashed line for the patchy epoch of model B with constant ionization efficiency, $\mathrm{z}=11-19$. The two histograms representing the earlier cosmological epochs are well-approximated by Gaussians.

anisotropies created during reionization one has to observe angular scales where photon diffusion smoothes out the primary anisotropies.

Upcoming experiments such as ACT and SPT will observe the sky in a number of frequency bands with comparable sensitivity and angular resolution. ACT (Kosowskv 2003) will observe in three frequency bands: 145,225 , and $265 \mathrm{GHz}$, with FWHM beam-widths $\theta_{F W H M}$ of $1.7,1.1$, and 0.9 arcminutes, respectively. We use the specifications of the $225 \mathrm{GHz}$ channel for our power spectrum analysis because the thermal SZ effect almost vanishes in this frequency regime, its zero being at $\nu \simeq 218 \mathrm{GHz}$. The sensitivity per resolution pixel for this channel is $\sigma=2 \mu K$. The South Pole Telescope (Ruhl et al. 2004) will observe in five bands at 95, 150, 219, 274, and $345 \mathrm{GHz}$. The $219 \mathrm{GHz}$ channel will have
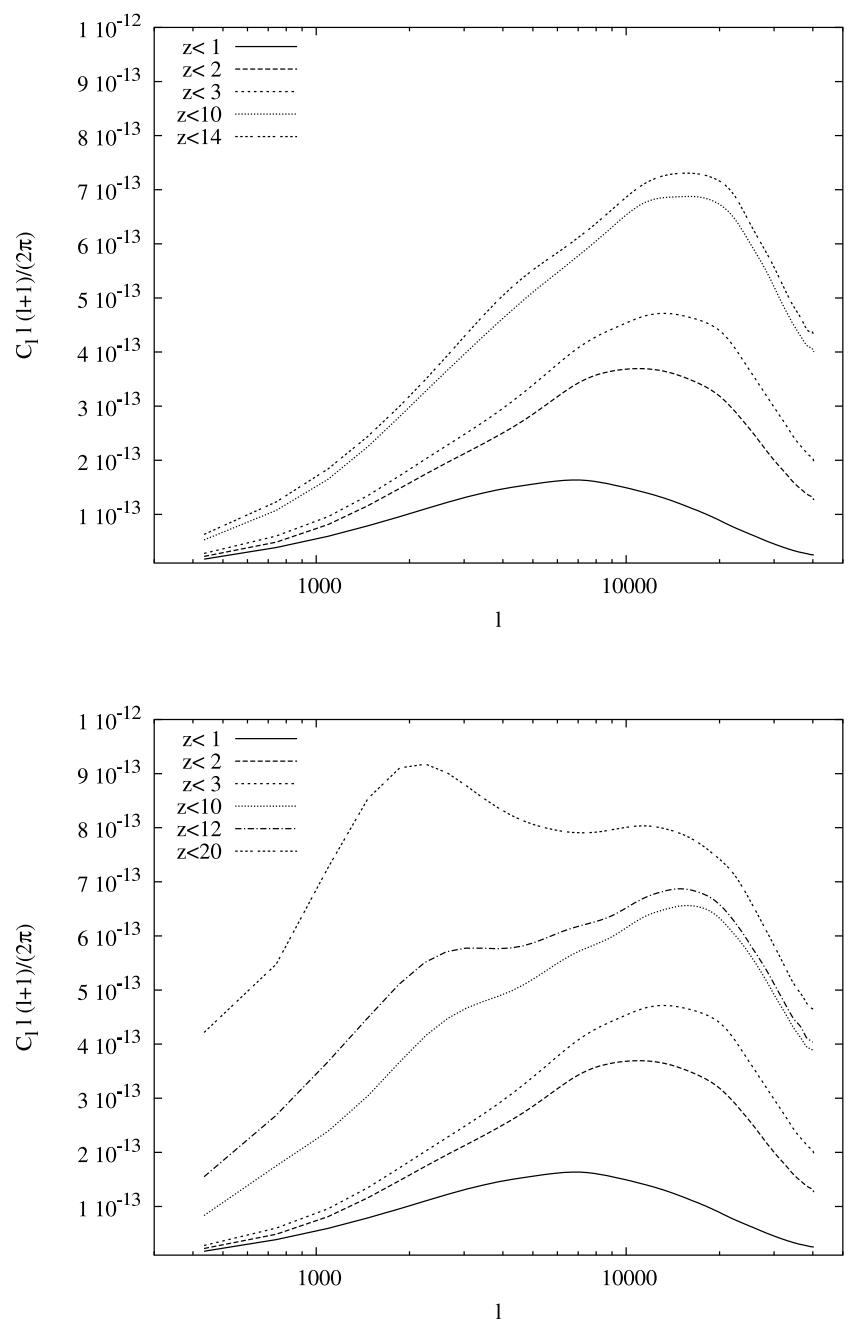

Fig. 6.- Contribution to the kinetic SZ signal for Models A (upper panel) and $\mathrm{C}$ (lower panel) out to different redshifts. Although a large fraction of the total Doppler effect comes from low redshift, high overdensity regions, there is a significant contribution out to $z \simeq 10$. In the patchy model (bottom) the regime $z=11-19$ leads to further enhancement of the signal. 


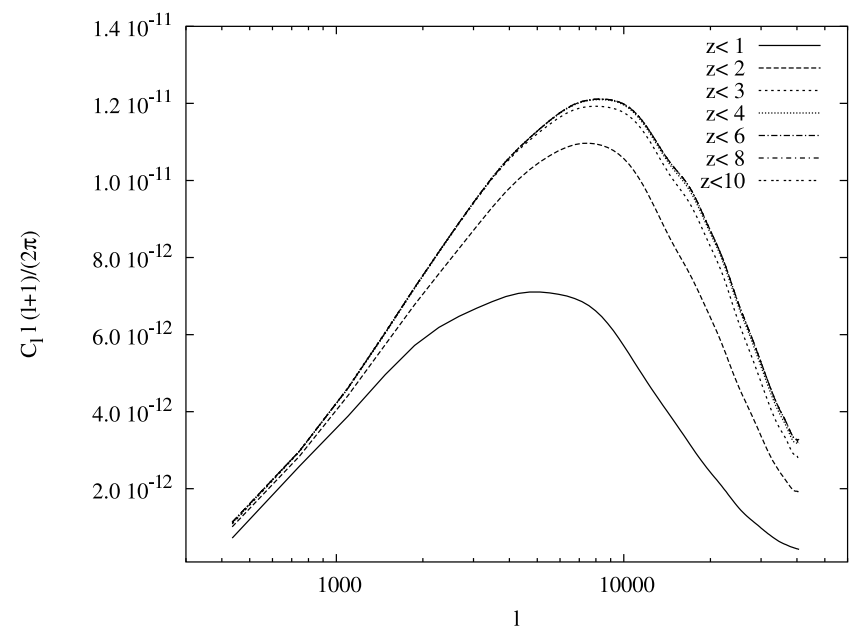

FIG. 7.- Redshift dependence of the thermal Sunyaev-Zel'dovich $\Delta T / T=-2 y$ effect as generated with our simulations. The major contribution comes from galaxy clusters in the regime $z<3$. Hence, the thermal SZ effect is less suited for studying the reionization epoch than is the kinetic SZ effect.

an angular resolution of $\theta_{F W H M}=0.69^{\prime}$ and a sensitivity of $10 \mu K^{8}$. We assume sky coverages of $0.5 \%$ and $10 \%$ for ACT and SPT, respectively.

From these specifications and a template for the power spectrum of primary and secondary anisotropies at arcminute scales (which we obtain from our simulations), we can calculate the errors in the $C_{l}$ determination, including noise as additional random field on the sky (Tegmark 1997)):

$$
\Delta C_{l}=\sqrt{\frac{2}{f_{s k y}(2 l+1)}}\left[C_{l}+\frac{f_{s k y}}{w B_{l}^{2}}\right],
$$

where we assume errors bars corresponding to a Gaussian map. Here, $w=\left(\theta_{f w h m} \sigma\right)^{-2}$, and $B_{l}$ is a beam profile (assumed Gaussian) given by $B_{l}=e^{-\theta_{b}^{2} l(l+1) / 2}$ (with $\theta_{b}=\theta_{f w h m} / \sqrt{8 \ln 2}$ ).

We assume that experiments such as ALMA ${ }^{9}$ will lead to a good understanding of the point source frequency spectrum and angular clustering. In more pessimistic scenarios where only the frequency dependence or nothing is known about point sources, they can strongly degrade our ability to detect the kinetic SZ (Huffenberger \& Seliak 2004) (we included their estimate for the IR source power spectrum at $145 \mathrm{GHz}$ in Figure 8). On the other hand the assumption of perfect cleaning of the thermal SZ effect is safer because we understand its frequency dependence well.

To compare the experimental constraints with the power spectra extracted from our simulations on degree patches on the sky, we bin the errors into bands of width $\Delta l=360$. The predicted measurements errors are plotted in Figure 9] together with power spectra generated from the "cleaned" maps of primordial CMB and kinetic

8 J.E. Ruhl, private communication.

9 http://www.eso.org/projects/alma/science/alma-science.pdf
SZ combined. Since the kinetic SZ appears almost featureless on the scales accessible to the next generation of experiments, reionization models can be distinguished by their average amplitude on these scales. The error bars in the relevant band-powers of ACT are combined using $\sigma_{\text {tot }}^{2}=\left(\sum_{i} \sigma_{i}^{-2}\right)^{-1}$. It follows that the overall amplitude can be measured with an accuracy of $\sigma_{\Delta T}=0.011 \mu \mathrm{K}$ (we assumed a $1 \%$ calibration uncertainty). Given that the plateau of the extended patchy model (Model C) lies at $\Delta T \simeq 2.407 \mu \mathrm{K}$, while Model $\mathrm{A}$ has an average amplitude on these scales of $\Delta T \simeq 2.076 \mu K$, the two will be distinguishable at the $30 \sigma$ level with ACT, if we ignore contamination by point sources and by the thermal SZ, which is not a realistic assumption. This is the same for the South Pole Telescope, which has better angular resolution, but will look less deep (it has a much larger survey area).

It is also of interest to ask whether additional constraints on reionization scenarios could be achieved by using non-Gaussian statistics, in particular the four point function (the skewness arising from a line-of-sight velocity effect should vanish). It may be expected, that because the HII regions are created inside large overdensities at high redshift, patchy models are more Gaussian on the whole. The main problem with this notion is that in any patchy model with a comoving bubble size comparable to ours this effect will suffer from "washing out" by the primordial CMB. On scales where Doppler induced fluctuations become larger than the primordial CMB, the impact of patchiness amounts only to a fraction of the total signal (15\% in Model B, 30\% in Model C), the rest being attributed to scatterings owing to density modulations alone. Also, the majority of non Gaussian contributions comes from clusters and filaments at $z<3$, compare Figure [5] We computed the kurtosis

$$
\Theta_{4} \equiv \frac{\left\langle(\Delta T / T)^{4}\right\rangle}{\sigma_{T}^{4}}-3
$$

of thermal SZ "cleaned" maps (i.e. primordial $\mathrm{CMB}+$ Doppler) that were filtered with a Gaussian in Fourier space, approximately cutting out "contamination" by the primordial CMB anisotropies below $l \simeq$ 3500 , and beam smearing at scales of $l \simeq 9000$ comparable to the angular resolution of ACT (similar to the window function proposed by Huffenberger \& Seliak 2004). Using this method, we do not find a statistically significant difference between patchy and homogeneous models of reionization.

\subsection{Expected bias in cosmological parameter determination from Planck}

Cosmic microwave background measurements have been and will likely remain the most precise tools for the measurement of cosmological parameters. The best constraints will come from a combination of CMB temperature and polarization power spectra which encapsulate all the relevant information in the sky maps. The aim is to make the data cosmic variance limited to as high as possible multipole numbers. WMAP is cosmic variance limited up to $l \simeq 500$.

The Planck satellite should achieve cosmic variance limitation out to $l=2500$ for its temperature power spectrum measurement. Hence, Planck will reach into 


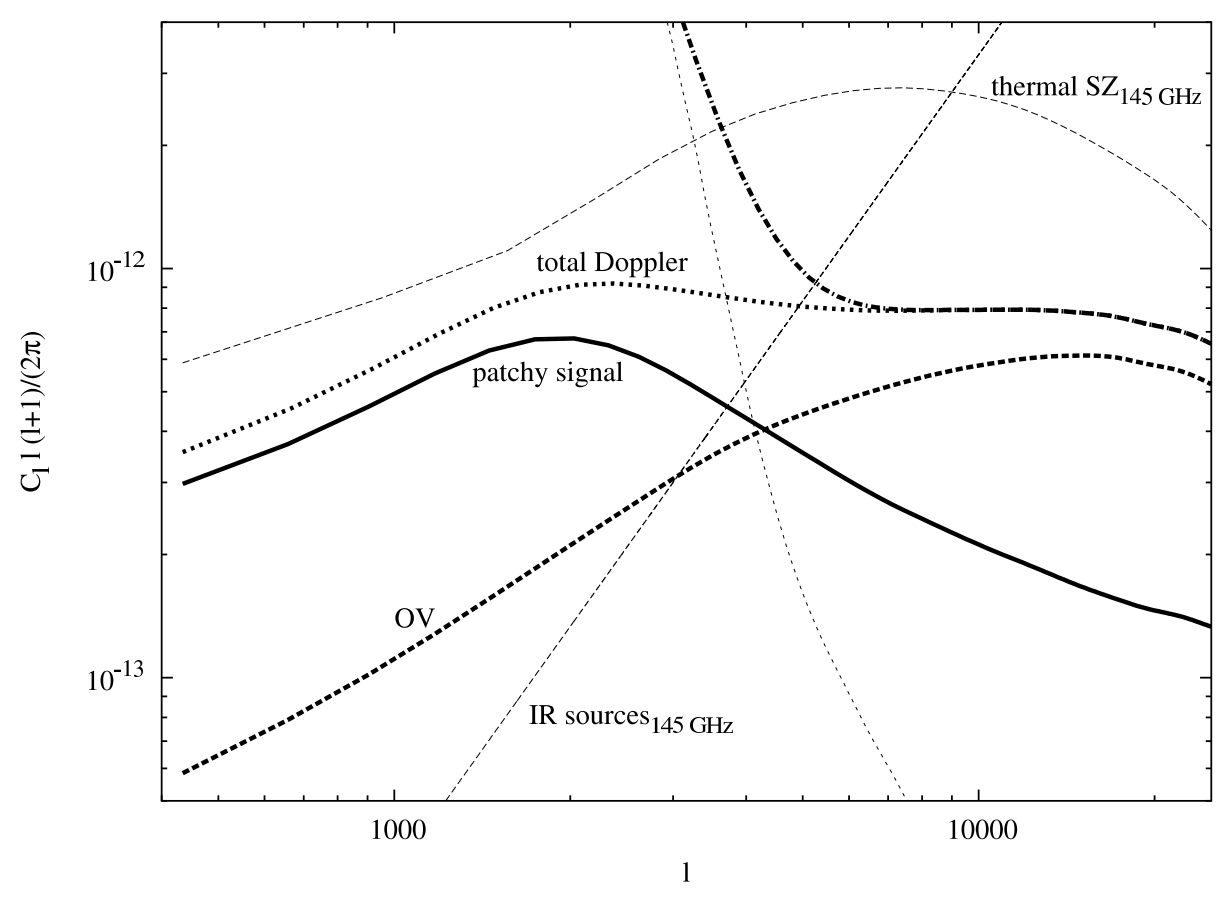

FIG. 8.- Different contributions to the total Doppler signal (kinetic SZ) in our extended patchy reionization Model C. The solid curve is the contribution to temperature anisotropies from the patchy regime alone at $z=7-20$. The dashed curve gives the Doppler effect from density modulations at homogeneous ionization out to $z=11$. The dotted curve sums up those contributions to the total Doppler signal. The total temperature fluctuations, cleaned of the thermal SZ and IR sources (shown at $145 \mathrm{GHz}$ in the thin dashed lines), is given by the dot-dashed curve. These curves are smoothed versions of the power spectra generated from 50 maps.

the regime where the secondary anisotropies become important. To avoid biases in parameter estimation, systematic changes that the secondaries may produce in the power spectra need to be considered.

The contribution to the power spectrum from patchy reionization is one to two orders of magnitude smaller than the primordial CMB anisotropies on the relevant scales, but owing to the exquisite sensitivity of these experiments it biases parameter estimates. For the analytic models of patchy reionization suggested by Knox et al. (1998) and Santos et al. (2003) this parameter bias was estimated. In these models the mean bubble size is smaller than in our computation, and the signal peaks at higher multipoles.

The bias can be estimated from the Fisher matrix coefficients

$$
F_{i j}=\sum_{l} \frac{\partial C_{l}}{\partial p_{i}} w_{l} \frac{\partial C_{l}}{\partial p_{j}}
$$

in the following manner:

$$
\mathcal{B}_{i} \equiv \frac{\Delta p_{i}}{\sigma_{i}}=\frac{\sum_{j} F_{i j}^{-1} \sum_{l} \frac{\partial C_{l}}{\partial p_{j}} C_{l}^{\mathrm{D}}}{\sqrt{F_{i i}^{-1}}}
$$

where $\Delta p_{i}$ are the systematic biases in the determination of parameters $p_{i}, w_{l}$ are the inverse squares of the statistical errors in the $C_{l}$ estimation, given by Equation $[7$ and $\sigma_{i}=\sqrt{F_{i i}^{-1}}$ is the estimate of the error bars for parameter $i$. In this expression, $C_{l}^{\mathrm{D}}$ denotes the combined

\begin{tabular}{|c|c|c|c|c|c|c|}
\hline model & $\tau_{r i}$ & $\Omega_{\Lambda}$ & $\omega_{d m}$ & $\omega_{b}$ & $n_{s}$ & $A_{s}$ \\
\hline $1 \sigma$ & 0.0035 & 0.010 & 0.0017 & 0.00018 & 0.0045 & 0.0050 \\
\hline $\mathrm{A}$ & 0.40 & 1.18 & -1.10 & 1.71 & 2.14 & 0.16 \\
$\mathrm{~B}$ & 1.26 & 1.66 & -1.54 & 2.40 & 3.05 & 0.78 \\
$\mathrm{C}$ & 2.25 & 2.89 & -2.69 & 4.20 & 5.62 & 1.58 \\
\hline
\end{tabular}

TABLE 1

BiAs IN UNITS OF THE STATISTICAL ERROR $\left(\mathcal{B}_{i}\right)$ EXPECTED FOR COSMOlOgical PARAMETER ESTIMATION With PlancK, IF TEMPERATURE AND POLARIZATION POWER SPECTRA ARE USED AND THE INFLUENCES OF KSZ/OV AND PATCHY REIONIZATION ARE NEGLECTED IN THE POWER SPECTRUM ANALYSIS. The MAXIMUM MULTIPOLE IN OUR ANALYSIS WAS $l=4000$.

Doppler power spectrum owing to kSZ/OV and patchy reionization.

In Table 1 we show the results of our analysis, using the power spectra for our different models as bias ${ }^{10}$. The first line shows the statistical error alone, $\sqrt{F_{i i}^{-1}}$,

10 We combined the three frequency channels of Planck with the highest angular resolution and took into account the number of polarized instruments. For a one year observation period, the three channels $(217,143$ and $100 \mathrm{GHz})$ have $\theta_{\mathrm{fwhm}}=5.0,7.1$ and 9.2 arcminutes. This leads to the raw sensitivities:

$$
\begin{aligned}
& w_{T}^{-1}=(0.0084 \mu K)^{2} \\
& w_{P}^{-1}=(0.0200 \mu K)^{2} .
\end{aligned}
$$

We assumed a sky coverage of $f_{S k y}=0.8$. 


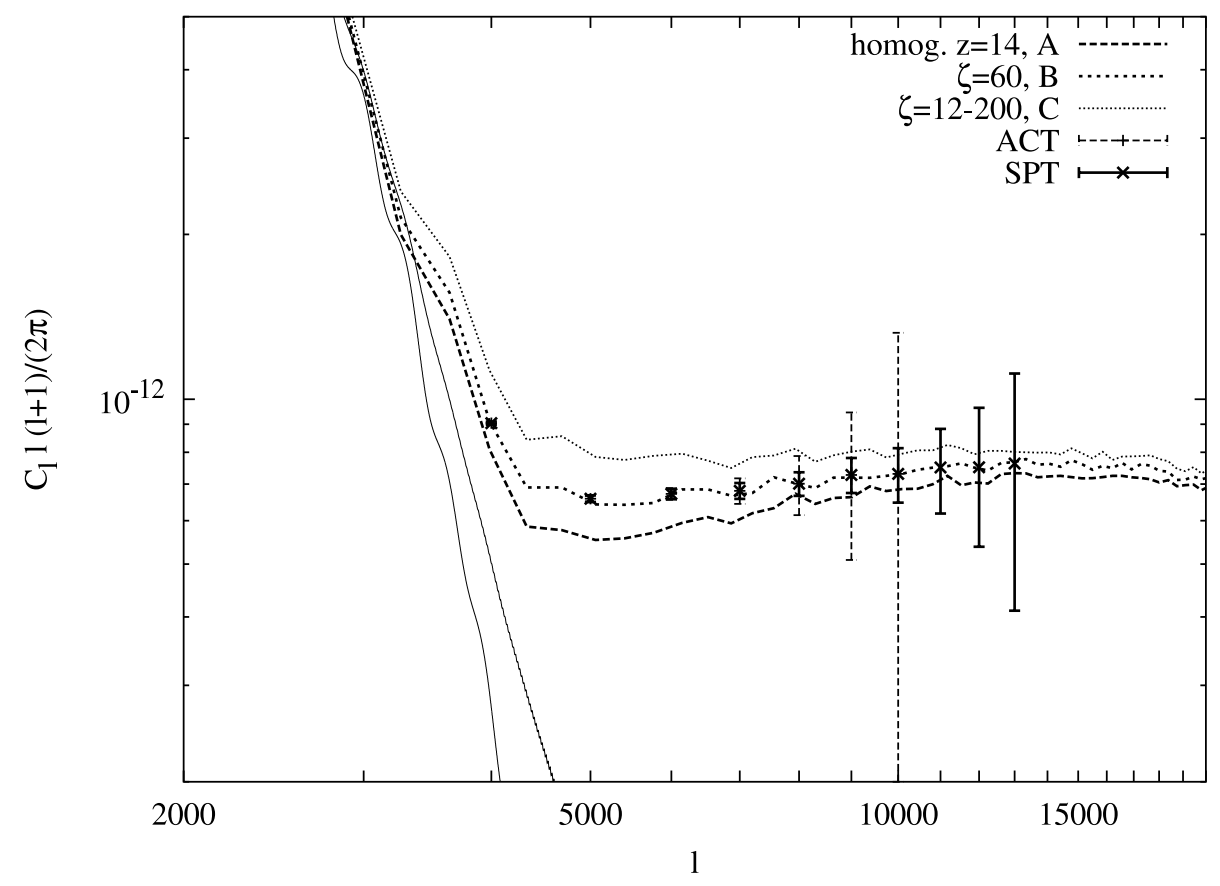

FIG. 9.- With future experiments like ACT, the various reionization models should be easily distinguishable by measuring the temperature power spectrum. Since the kinetic SZ signal is almost featureless on the scales of experimental relevance, the band-powers can be combined to a simple amplitude when distinguishing the different models. We find that the double reionization scenario (given by the upper power spectrum) could thus be distinguished at high significance from a uniform, instantaneous model where the universe reionizes at $z=14$ (bottom curve). The change of the ionizing efficiency with time $\dot{\zeta}$ affects the slope of the secondary anisotropy power spectra.

the following lines show the parameter bias $\mathcal{B}_{i}$ for Models A, B, and C. For Planck, we used the specifications of the High Frequency Instrument ${ }^{11}$. The power spectra derivatives for the Fisher analysis were computed for the fiducial cosmological model given in the introduction.

It is clear from Table 1 that secondary anisotropies need to be taken into account. This is the case in particular for parameters that influence the shape of the power spectra at intermediate scales $\left(\omega_{b}, \omega_{d m}, n_{s}\right)$. Constraints on the amplitude $A_{s}$ and the optical depth owing to reionization $\tau_{\text {ri }}$ are less heavily biased (besides the amplitude, the reionization optical depth only affects $\mathrm{CMB}$ polarization on large scales). Note that even in a simple homogeneous reionization model, as our Model A, most of the biases are of order unity. The individual systematic shifts depend strongly on what parameters are used. The analysis of Santos et al. (2003) has extra parameters that make the biasing source (the patchy power spectrum) distribute differently into each parameter offset.

The polarization anisotropies generated during the reionization epoch are expected to be four orders of magnitude smaller than the temperature power spectra $(\mathrm{Hu}$ 2000). This suggests that to avoid biases one could use temperature information down to an angular scale where the Doppler contamination is still negligible but use the polarization data for all $l$. We show the results of an analysis in which we used temperature information only

\begin{tabular}{|c|c|c|c|c|c|c|}
\hline model & $\tau_{r i}$ & $\Omega_{\Lambda}$ & $\omega_{d m}$ & $\omega_{b}$ & $n_{s}$ & $A_{s}$ \\
\hline $1 \sigma$ & 0.0041 & 0.013 & 0.0020 & 0.00026 & 0.0079 & 0.0056 \\
\hline $\mathrm{A}$ & 0.010 & -0.018 & 0.017 & 0.024 & 0.086 & 0.068 \\
$\mathrm{~B}$ & 0.027 & -0.028 & 0.026 & 0.037 & 0.135 & 0.196 \\
$\mathrm{C}$ & 0.050 & -0.070 & 0.067 & 0.058 & 0.301 & 0.487 \\
\hline
\end{tabular}

\section{TABLE 2}

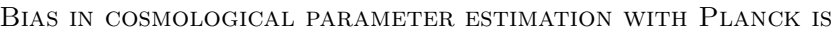
REDUCED SIGNIFICANTLY, WHEN TEMPERATURE POWER SPECTRA ARE USED ONLY UNTIL $l=1000$ AND POLARIZATION POWER SPECTRA ARE USED IN THE WhOle RANGe (Planck SHOUld BE ABLE TO MEASURE $C_{l}^{E E}$ OUT TO $l \simeq 1800$ ). ON THE OTHER HAND, THE PARAMETER ERRORS BECOME ONLY SLIGHTLY LARGER BY LEAVING OUT THE HIGH $l$ TEMPERATURE INFORMATION.

out to $l \simeq 1000$, but polarization in the full range accessible to Planck (this will be out to $l \simeq 1800$ ) in Table 2 The $1 \sigma$ error bars for the parameters are only slightly larger than in Table 1 indicating that the parameter estimates are more or less "saturated" at $l \simeq 1000$. On the other hand, the expected parameter biases owing to reionization are much smaller. The use of polarization information in future $\mathrm{CMB}$ experiments thus can play an important role beyond breaking degeneracies between traditional cosmological parameters and improving the error bars.

The other strategy is to include an extra parameter to model the effect of the Doppler contributions. This

\footnotetext{
${ }^{11} \mathrm{http}$ ///www.rssd.esa.int/index.php?project=PLANCK\&page=perf_tqpproach has the added advantage that a positive con-
} 
straint on reionization could be obtained with Planck alone. Patchiness shifts the power spectrum on small scales, and we model this by a "patchy amplitude" parameter. This parameter is included by adding the Doppler spectra given in the last section with a variable amplitude $A_{D}$ to the primordial CMB. If the parameter derivatives in the Fisher matrix center $\left(A_{D}=1\right)$ around Model B, we find that the amplitude parameter could be constrained with $\sigma_{A_{D}}=0.51$. The model is only $\simeq 0.3 \sigma$ away from homogeneous reionization (Model $\mathrm{A}$ ), so Planck will not be able to make a strong statement. If patchy reionization turns out to be extended, similar to Model C, Planck should observe this at higher significance $\sigma_{A_{D}}=0.20$, amounting to a $5 \sigma$ detection of Doppler induced secondary anisotropies, with the amplitude of Model $\mathrm{C}$ being $3 \sigma$ away from homogeneous reionization. Finally, if reionization proceeded homogeneously, Planck will not gain knowledge beyond its large scale polarization measurements of $\tau_{r i}$ by using small scale temperature fluctuations, given that the uncertainty for our Model A lies at $\sigma_{A_{D}}=0.71$.

When Planck's power spectra are modeled with the "patchy amplitude" parameter, constraints for the standard cosmological parameters are slightly improved over the analysis that abandoned temperature data beyond $l=1000$ (Table 2), despite the introduction of an extra parameter. Concretely, we find in this analysis that $\sigma_{\tau_{r i}}=0.037, \sigma_{\Omega_{\Lambda}}=0.011, \sigma_{\omega_{d m}}=0.0018$, $\sigma_{\omega_{b}}=0.00021, \sigma_{\Omega_{n_{s}}}=0.0058, \sigma_{A_{s}}=0.0052$.

The Doppler power spectrum can bias the result of parameter analyses with future CMB surveys. Planck may be able to improve constraints on models with an extended epoch of patchiness. We hope to have shown in this section that careful modeling of the epoch of first stars will become crucial for doing precision cosmology with progressively more refined CMB experiments.

\section{DISCUSSION}

We have presented simulations of secondary anisotropies in the cosmic microwave background calculated using smoothed particle hydrodynamics simulations of large scale structure, focusing on the effect produced by patchy reionization. We incorporated an analytic model for the morphology of the HII regions into our numerical treatment and investigated whether such patchy scenarios can be distinguished from homogeneous reionization.

An important advantage of our technique over pure analytical predictions of patchy reionization morphology is that we follow the complicated clustering of dark matter and baryons into the slightly non-linear regime. In contrast to full radiative transfer calculations of the reionization epoch, we combine uncertainties in the physics of source formation, feedback processes and radiative transfer into a single parameter and explore the consequences of varying this parameter. This simplification allows us to make predictions on scales an order of magnitude larger than current radiative transfer schemes can accomplish with a small expense of memory and CPU.

We extracted power spectra from sky maps produced by tracing rays across our simulation volumes. The patchy reionization signal peaks on multipole scales of $l \simeq 2000$, and it increases the amplitude of the "cleaned" CMB power spectrum by up to $30 \%$ on scales $l \geq 4000$, so that the total level of Doppler related anisotropies is $\Delta T \simeq 2.4 \mu \mathrm{K}$. We found that with the next generation of ground based CMB experiments (ACT, SPT) the different reionization models we investigated could be distinguished with high significance by using the power spectrum. Additional information about the morphological properties of that epoch that could be obtained for instance by measuring the four point function or other deviations from Gaussianity will probably be difficult to obtain, because the reionization signal peaks on angular scales where the primordial CMB anisotropies dominate. In the future, it may be possible to combine measurements of the CMB with other observations such as $21 \mathrm{~cm}$ fluctuations from neutral hydrogen (Zaldarriaga et al. 2004; Coorav 2004) or Lyman-alpha emission from high redshift galaxies (e.g. Furlanetto et al. 2004d) to further constrain the topology of the reionization process.

We investigated the bias in the determination of cosmological parameters that will be produced by the additional patchy reionization signal, when extracting cosmological parameters from CMB anisotropy measurements. We find that for Planck this bias is significant. The bias may be circumvented by focusing completely on polarization information in the multipole regime where patchiness peaks, with only a slight disadvantage in parameter constraints. Alternatively, a template for the Doppler spectrum could be introduced in the parameter analysis which may lead to a detection of the effects of an extended reionization phase.

O. Z. and M. Z. are supported by NSF grant AST 0098606 and by the David and Lucille Packard Foundation Fellowship for Science and Engineering and by the Sloan Foundation. This work is also supported by NSF grants ACI 96-19019, AST 00-71019, AST 02-06299, and AST 03-07690, and NASA ATP grants NAG5-12140, NAG5-13292, and NAG5-13381. The simulations were performed at the Center for Parallel Astrophysical Computing at the Harvard-Smithsonian Center for Astrophysics.

\section{REFERENCES}

Abel, T. \& Wandelt, B. D. 2002, MNRAS, 330, L53

Barkana, R. \& Loeb, A. 2001, Phys. Rep., 349, 125

Barkana, R. \& Loeb, A. 2004, ApJ, 609, 474

Bartelmann, M. \& Schneider, P. 2001, Phys. Rept., 340, 291

Becker, R. H. et al. 2001, AJ, 122, 2850

Bennett, C. et al. 2003, ApJS, 148, 97

Birkinshaw, M. 1999, Phys. Rept., 310, 97

Bond, J. R., Cole, S., Efstathiou, G., \& Kaiser, N. 1991, ApJ, 379, 440
Bond, J. R., Contaldi, C. R., \& Pogosyan, D. 2003, Phil. Trans. Roy. Soc. Lond., A361, 2435

Bromm, V., Coppi, P. S., \& Larson, R. B. 2002, in Lighthouses of the Universe: The Most Luminous Celestial Objects and Their Use for Cosmology Proceedings of the MPA/ESO/, p. 316, 316$+$

Carlstrom, J. E., Joy, M. K., Grego, L., Holder, G. P., Holzapfel, W. L., Mohr, J. J., Patel, S., \& Reese, E. D. 2000, Physica Scripta Volume T, 85, 148

Cen, R. 2003, ApJ, 591, L5 
Ciardi, B., Ferrara, A., \& White, S. D. M. 2003, MNRAS, 344, L7 Cooray, A. 2004, Phys. Rev. D 70, 063509

de Oliveira-Costa, A., Tegmark, M., Zaldarriaga, M., \& Hamilton, A. 2004, Phys. Rev., D69, 063516

Fan, X.-H. et al. 2001, AJ, 122, 2833

Fan, X.-H. et al. 2004, AJ, 128, 515

Furlanetto, S. \& Loeb, A. 2004, ApJ, in press astro-ph/0409656

Furlanetto, S., Sokasian, A., \& Hernquist, L. 2004a, MNRAS, 347, 187

Furlanetto, S., Zaldarriaga, M., \& Hernquist, L. 2004b, ApJ, in press astro-ph/0403697.

Furlanetto, S., Zaldarriaga, M., \& Hernquist, L. 2004c, ApJ, in press astro-ph/0404112

Furlanetto, S., Hernquist, L., \& Zaldarriaga, M. 2004d, ApJ, in press astro-ph/0406131

Gnedin, N. Y. \& Jatte, A. H. 2001, ApJ, 551, 3

Gruzinov, A. \& Hu, W. 1998, ApJ, 508, 435

Gunn, J. E. \& Peterson, B. A. 1965, ApJ, 142, 1633

Holzapfel, W. L. et al. 1999, Bulletin of the American Astronomical Society, 34, 707

Hu, W. 2000, ApJ, 529, 12

—. 2002, Phys. Rev., D65, 023003

Hu, W. \& Holder, G. P. 2003, Phys. Rev., D68, 023001

Hu, W. \& Okamoto, T. 2002, ApJ, 574, 566

Huffenberger, K. M. \& Seljak, U. 2004

Hui, L. \& Haiman, Z. 2003, ApJ, 596, 9

Kaplinghat, M., Knox, L., \& Song, Y.-S. 2003, Phys. Rev. Lett., 91, 241301

Knox, L., Scoccimarro, R., \& Dodelson, S. 1998, Phys. Rev. Lett., 81,2004

Kogut, A. et al. 2003, ApJS, 148, 161

Kosowsky, A. 2003, New Astron. Rev., 47, 939

Lewis, A., Challinor, A., \& Lasenby, A. 2000, ApJ, 538, 473

Mackey, J., Bromm, V. \& Hernquist, L. 2003, ApJ, 586, 1

Metcalf, R. B. \& Silk, J. 1998, ApJ, 492, L1+

Miralda-Escudé, J., Haehnelt, M., \& Rees, M. J. 1998, ApJ, 530, 1

Ostriker, J. \& Vishniac, E. 1986, ApJ, 306, 51

Readhead, A. C. S. et al. 2004, ApJ, 609, 498

Ruhl, J.et al. 2004, in Astronomical Structures and Mechanisms Technology. Edited by Antebi, Joseph; Lemke, Dietrich. Proceedings of the SPIE, Volume 5498, pp. 11-29 (2004)., 1129
Santos, M. G., Cooray, A., Haiman, Z., Knox, L., \& Ma, C.-P. 2003, ApJ, 598, 756

Schaerer, D. 2003, A\&A, 397, 527

Seljak, U. \& Zaldarriaga, M. 1996, ApJ, 469, 437

Seljak, U. et al. 2004

Sokasian, A., Abel, T., \& Hernquist, L. 2002, MNRAS, 332, 601

Sokasian, A., Abel, T., Hernquist, L., \& Springel, V. 2003, MNRAS, 344,607

Sokasian, A., Yoshida, N., Abel, T., Hernquist, L., \& Springel, V. 2004, MNRAS, 350, 47

Somerville, R. S., Bullock, J.S. \& Livio, M. 2003, ApJ, 593, 611

Spergel, D. N. et al. 2003, ApJS, 148, 175

Springel, V. \& Hernquist, L. 2002, MNRAS, 333, 649

Springel, V. \& Hernquist, L. 2003, MNRAS, 339, 289

Springel, V., Yoshida, N. \& White, S.D.M. 2001, NewA, 6, 79

Stompor, R. \& Efstathiou, G. 1999, MNRAS, 302, 735

Sunyaev, R. A. \& Zel'dovich, Y. B. 1980, MNRAS, 190, 413

Tegmark, M. 1997, Phys. Rev., D56, 4514

Tegmark, M. et al. 2004, Phys. Rev., D69, 103501

Theuns, T. et al. 2002, ApJ, 567, L103

Van Waerbeke, L., Bernardeau, F., \& Benabed, K. 2000, ApJ, 540, 14

White, M. J., Hernquist, L., \& Springel, V. 2002, ApJ, 579, 16

White, R. L., Becker, R. H., Fan, X.-H., \& Strauss, M. A. 2003, AJ, 126, 1

Wyithe, J. S. B. \& Loeb, A. 2003, ApJ, 588, L69

Yoshida, N., Abel, T., Hernquist, L., \& Sugiyama, N. 2003,ApJ, 592,645

Yoshida, N., Bromm, V. \& Hernquist, L. 2004, ApJ, 605, 579

Zaldarriaga, M. 1997, Phys. Rev., D55, 1822

Zaldarriaga, M. \& Seljak, U. 1999, Phys. Rev., D59, 123507

Zaldarriaga, M., Furlanetto, S., \& Hernquist, L. 2004, ApJ, 608, 622

Zel'dovich, Y. B. \& Sunyaev, R. A. 1969, Astrophys. Space Sci., 4, 301 